\title{
Potentiation of ghrelin signaling attenuates cancer anorexia-cachexia and prolongs survival
}

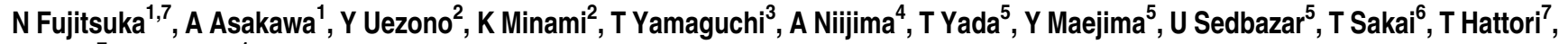 \\ $Y \mathrm{Kase}^{7}$ and $A$ Inui ${ }^{1}$
}

Cancer anorexia-cachexia syndrome is characterized by decreased food intake, weight loss, muscle tissue wasting and psychological distress, and this syndrome is a major source of increased morbidity and mortality in cancer patients. This study aimed to clarify the gut-brain peptides involved in the pathogenesis of the syndrome and determine effective treatment for cancer anorexia-cachexia. We show that both ghrelin insufficiency and resistance were observed in tumor-bearing rats. Corticotropin-releasing factor (CRF) decreased the plasma level of acyl ghrelin, and its receptor antagonist, $\alpha$-helical CRF, increased food intake of these rats. The serotonin 2c receptor (5-HT2CR) antagonist SB242084 decreased hypothalamic CRF level and improved anorexia, gastrointestinal (Gl) dysmotility and body weight loss. The ghrelin receptor antagonist (D-Lys3)GHRP-6 worsened anorexia and hastened death in tumor-bearing rats. Ghrelin attenuated anorexia-cachexia in the short term, but failed to prolong survival, as did SB242084 administration. In addition, the herbal medicine rikkunshito improved anorexia, GI dysmotility, muscle wasting, and anxiety-related behavior and prolonged survival in animals and patients with cancer. The appetite-stimulating effect of rikkunshito was blocked by (D-Lys3)-GHRP-6. Active components of rikkunshito, hesperidin and atractylodin, potentiated ghrelin secretion and receptor signaling, respectively, and atractylodin prolonged survival in tumorbearing rats. Our study demonstrates that the integrated mechanism underlying cancer anorexia-cachexia involves lowered ghrelin signaling due to excessive hypothalamic interactions of 5-HT with CRF through the 5-HT2cR. Potentiation of ghrelin receptor signaling may be an attractive treatment for anorexia, muscle wasting and prolong survival in patients with cancer anorexia-cachexia.

Translational Psychiatry (2011) 1, e23; doi:10.1038/tp.2011.25; published online 26 July 2011

\section{Introduction}

Cachexia is characterized by weight loss, fat and muscle tissue wasting, psychological distress and a lower quality of life. In cancer patients, anorexia development is frequently associated with the presence of cachexia, resulting in the socalled cancer anorexia-cachexia syndrome. ${ }^{1}$ This syndrome is observed in $80 \%$ of patients with advanced-stage cancer and is a frequent cause of death. ${ }^{2}$ Recent reports have indicated that an imbalance between anorexigenic and orexigenic peptides leads to appetite suppression. ${ }^{3-5}$ Anorexia-cachexia is caused predominantly by cytokines that are either produced by cancer cells or released by the host immune system in response to the cancer, ${ }^{6}$ but the neurochemical mechanisms responsible for cancer anorexia-cachexia remain uncertain. The two major options for pharmacological therapy are megestrol acetate and glucocorticoids, ${ }^{7,8}$ but both have limited effectiveness. A better understanding of the underlying mechanisms of this syndrome will help in the development of new therapies to improve quality of life and potentially to prolong survival in patients with cancer-induced anorexia-cachexia.

Anxiety and depressive symptoms are associated with various gastrointestinal (Gl) disorders, including cancers, ${ }^{6}$ chronic liver diseases, inflammatory bowel diseases and functional Gl diseases. ${ }^{9,10}$ Corticotropin-releasing factor (CRF) is a mediator of the endocrine, autonomic and immune responses to stress, including anorexia and anxiety-related behavior. ${ }^{11}$ The central serotonin $(5-\mathrm{HT})$ system has also been implicated in the processes of meal satiation and satiety. Hypothalamic 5-HT and CRF activities are stimulated by proinflammatory cytokines in the circulation and the hypothalamus. $^{12}$ Therefore, we hypothesized that $5-\mathrm{HT}$ and CRF might have a role in the pathogenesis of cancer anorexiacachexia by modulating central and peripheral mechanisms as part of the stress response.

Ghrelin system is involved in eliciting feeding, inducing adiposity, and regulating glucose metabolism and body weight. $^{13}$ Ghrelin has an important role in triggering the adaptive response to starvation. In this study, we demonstrate

\footnotetext{
${ }^{1}$ Department of Psychosomatic Internal Medicine, Kagoshima University Graduate School of Medical and Dental Sciences, Kagoshima, Japan; ${ }^{2}$ Cancer Pathophysiology Division, National Cancer Center Research Institute, Tokyo, Japan; ${ }^{3}$ Division of Gastroenterology, Chiba Cancer Center, Chiba, Japan; ${ }^{4}$ Department of Physiology, Niigata University School of Medicine, Niigata, Japan; ${ }^{5}$ Department of Physiology, Jichi Medical University School of Medicine, Tochigi, Japan; ${ }^{6}$ Division of Life Science, Graduate School of Science and Engineering, Saitama University, Saitama, Japan and ${ }^{7}$ Tsumura Research Laboratories, Ibaraki, Japan Correspondence: Dr A Asakawa, Department of Psychosomatic Internal Medicine, Kagoshima University Graduate School of Medical and Dental Sciences, 8-35-1 Sakuragaoka, Kagoshima, 890-8520, Japan.

E-mail: asakawa@m2.kufm.kagoshima-u.ac.jp

Keywords: anorexia-cachexia; CRF; ghrelin; GHS-R; neuropeptide $Y$

Received 21 April 2011; revised 23 May 2011; accepted 16 June 2011
} 
that cancer anorexia-cachexia is mediated by decreased ghrelin signaling due to excessive hypothalamic interactions of 5-HT and CRF through the 5- $\mathrm{HT} 2 \mathrm{c}$ receptor $(5-\mathrm{HT} 2 \mathrm{cR})$ in a tumor-bearing rat model.

\section{Materials and methods}

Male Wistar rats were intraperitoneally (i.p.) inoculated with $\mathrm{AH}-130$ ascites hepatoma cells (Tohoku University, Sendai, Japan). The effects of $\alpha$-helical CRF, 5-HT2cR antagonist, ghrelin, ghrelin receptor (GHS-R) antagonist, and rikkunshito on food intake, weight and GI motility were examined in the tumor-bearing rats. Anxiety-related behavior was estimated using the open-field test. Plasma levels of peptides were determined by enzyme immunoassay. $\mathrm{Ca}^{2+}$ imaging and radioligand binding were performed using GHS-R-expressing cells and rat single neurons isolated from the arcuate nucleus $(\mathrm{ARC})$ or paraventricular nucleus (PVN). In all, 39 patients who had pathologically proven stage III/IV pancreatic cancer with ascites were eligible candidates for rikkunshito, as suggested from clinical experiences of this drug in Japan. The patients were retrospectively analyzed from 2004 to 2009 in Chiba Cancer Center (Chiba, Japan). To assess the difference among groups, a Student $t$-test or a multi-group Dunnett test was performed. Mortality data were compared using Kaplan-Meier plots and Gehan-Breslow-Wilcoxon tests (see Supplementary Materials).

\section{Results}

Ghrelin and cancer anorexia-cachexia. Decreased food intake, low rectal temperature, weight loss and wasting of muscle and fat mass were observed after tumor injection in rats (Figure 1a). Plasma concentrations of cytokines and c-reactive protein (CRP) were elevated (Figure 1b). Plasma acyl ghrelin concentrations were higher in tumor-bearing rats than in free-fed normal rats, but were significantly lower than in pair-fed normal rats and had an inverse relationship with plasma leptin concentrations (Figure 1c). Significant decreases in the hypothalamic expression of appetiteregulating peptides, neuropeptide $\mathrm{Y}$ (NPY), agouti-related peptide, proopiomelanocortin (POMC), urocortin-2,3 and $\mathrm{CRF}$, were observed in tumor-bearing rats compared to pair-fed controls (Figure 1d). This indicates a pathogenetic role of orexigenic peptides in cancer anorexia-cachexia.

Intravenous administration of ghrelin increased food intake for $2 \mathrm{~h}$, but not $6 \mathrm{~h}$ in normal and tumor-bearing rats on day 5 (Figure 1e). These responses were attenuated in tumorbearing rats compared with normal rats owing to ghrelin resistance. In contrast, i.p. administration of the GHS-R antagonist (D-Lys3)-GHRP-6 ( $4 \mu \mathrm{mol} \mathrm{kg}{ }^{-1}$; data not shown) worsened anorexia in tumor-bearing rats. Oral (per os) administration of a 5-HT2cR antagonist, SB242084 $\left(5 \mathrm{mg} \mathrm{kg}^{-1}\right)$, increased food intake in tumor-bearing rats (Figure 1f). The traditional herbal medicine rikkunshito, which stimulates the secretion of endogenous acyl ghrelin by blocking 5-HT2 receptors in rats, ${ }^{14}$ also increased food intake in tumor-bearing rats (Figure $1 \mathrm{~g}$ ). The effect of rikkunshito was inhibited by intravenous administration of (D-Lys3)-GHRP-6 $\left(2 \mu \mathrm{mol} \mathrm{kg}{ }^{-1}\right)$, suggesting mediation by endogenous acyl ghrelin. Daily administration of SB242084 or rikkunshito in tumor-bearing rats inhibited weight loss without affecting ascites volume (Supplementary Figure S1).

Phase III-like contractions in the antrum and duodenum of normal fasted rats are mediated by orexigenic signaling from ghrelin. ${ }^{15}$ Tumor-bearing rats exhibited fed-like motor activities in the antrum and duodenum, and the frequency of their phase III-like contractions significantly decreased (Supplementary Figure S2). Intravenous administration of ghrelin $(3 \mathrm{nmol})$ to tumor-bearing rats on day 5 immediately potentiated the fasted motor activity and increased the frequency of the phase III-like contractions (Figure 1h). Oral administration of SB242084 $\left(1 \mathrm{mg} \mathrm{kg}^{-1}\right)$ or rikkunshito $\left(1000 \mathrm{mg} \mathrm{kg}^{-1}\right)$ gradually restored the fasted motor patterns (Supplementary Figure S2).

Involvement of CRF in cancer anorexia. The cytosolic $\mathrm{Ca}^{2+}$ concentration $\left(\left[\mathrm{Ca}^{2+}\right]_{\mathrm{i}}\right)$ in single neurons isolated from the PVN of rats was measured by fura-2 microfluorometry. Administration of $10^{-5} \mathrm{moll}^{-1} 5-\mathrm{HT}$ for $10-15 \mathrm{~min}$ into superfusion solutions increased the $\left[\mathrm{Ca}^{2+}\right]_{i}$ in a continuous oscillatory manner. The $5-\mathrm{HT}$-induced $\left[\mathrm{Ca}^{2+}\right]_{i}$ increase was inhibited by administration of $100 \mathrm{\mu g} \mathrm{m}^{-1}$ rikkunshito to the PVN neurons; $83 \%$ of which subsequently demonstrated immunoreactivity to CRF (Figure 2a). In contrast, rikkunshito had little inhibitory effect on $30 \mathrm{mmol}^{-1}$ potassium chlorideinduced increases in $\left[\mathrm{Ca}^{2+}\right]_{i}$ (data not shown).

A significant decrease in the plasma concentration of acyl ghrelin was observed $3 \mathrm{~h}$ after intracerebroventricular administration of CRF $(1.5 \mathrm{nmol})$ to fasted rats (Figure $2 b)$, suggesting that endogenous ghrelin secretion is regulated by central CRF neurons. The electrophysiological study demonstrated that ghrelin and rikkunshito influenced CRFregulated adrenal function by decreased adrenal sympathetic nerve activity (Figure 2c).

Administration of a CRF antagonist, $\alpha$-helical CRF $(50 \mu \mathrm{g}$, intracerebroventricular), increased food intake in tumorbearing rats (Figure $2 \mathrm{~d}$ ), suggesting that the hypothalamic CRF system is activated in tumor-bearing rats, despite the overall reduction in CRF expression due to negative feedback inhibition resulting from increased corticosterone secretion (Figure 2e). CRF levels in the hypothalamus of tumor-bearing rats were significantly decreased by SB242084 and rikkunshito (Figure 2f). CRF-treated animals are known to display anxiety-related responses with decreased exploratory behavior. ${ }^{16}$ Tumor-bearing rats showed a significant decrease in rearing in the open-field test and increased fecal pellet output. Oral administration of rikkunshito to these rats recovered rearing and reversed fecal pellet output (Figure 2g).

Ghrelin signaling and rikkunshito. The afferent activity of the gastric vagus nerve decreased with intravenous administration of ghrelin (Figure 3a), as we have reported previously. ${ }^{17}$ In contrast, the efferent activities of the gastric (Figure $3 \mathrm{~b}$ ) and celiac (data not shown) branches of the vagus nerve increased with intravenous administration of ghrelin (10 ng). Similar effects were observed with intraduodenal, but not intragastric, administration of 

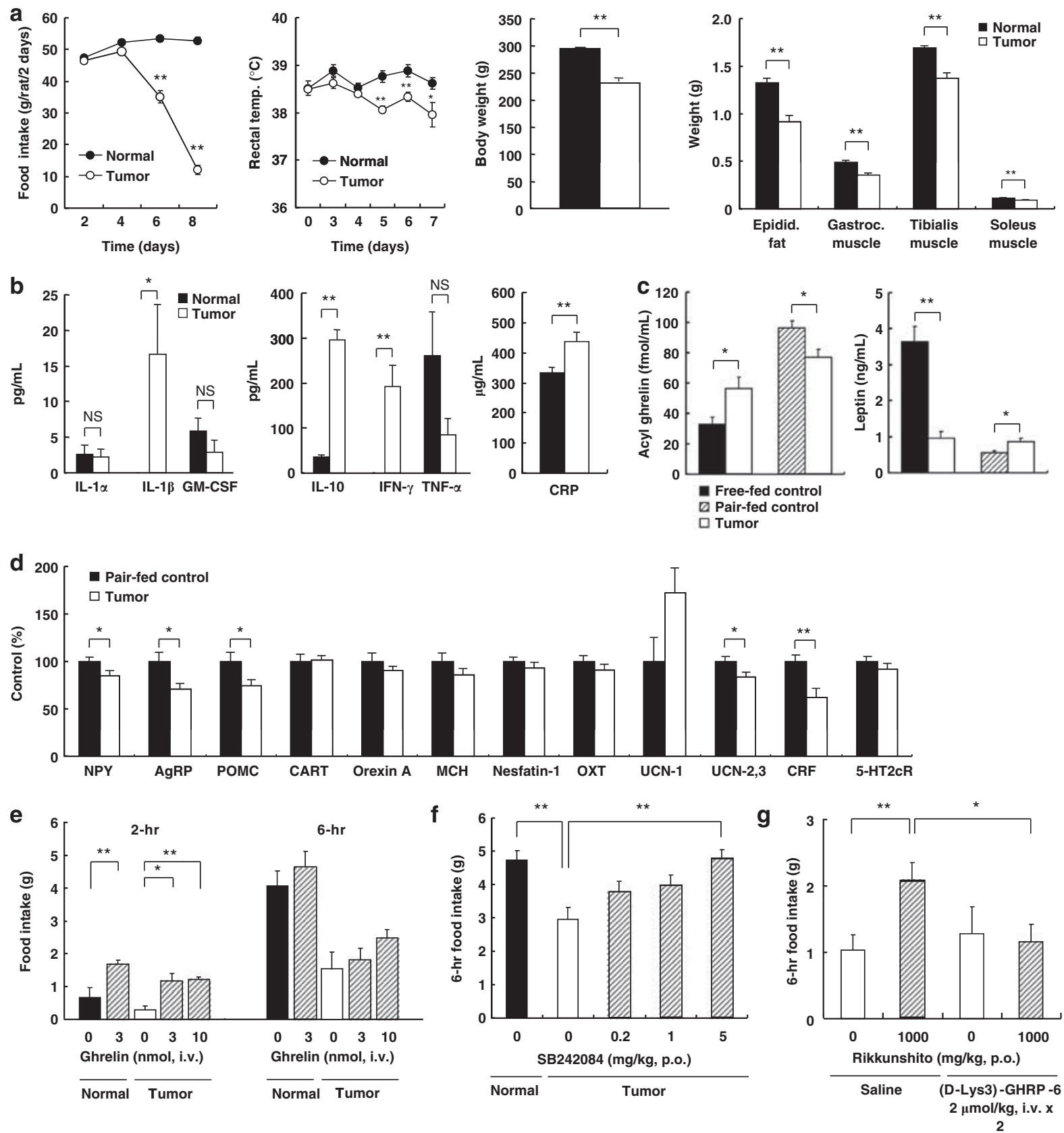

h
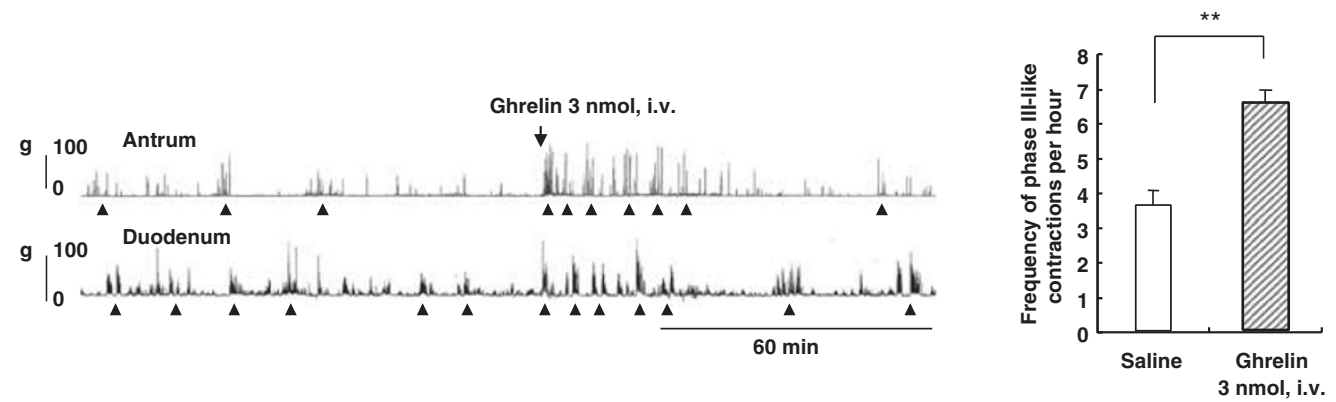

Figure 1 Cancer anorexia-cachexia. (a) Food intake, rectal temperatures and weights of tumor-bearing rats $(n=6-8)$. (b-d) Plasma and hypothalamic appetite-regulating peptides $(n=8-10)$. (e-g) Effects of ghrelin, the serotonin $2 \mathrm{c} \mathrm{receptor}(5-\mathrm{HT} 2 \mathrm{cR})$ antagonist SB242084 and rikkunshito on food intake of tumor-bearing rats and blockade by the ghrelin receptor (GHS-R) antagonist (D-Lys3)-GHRP-6 $(n=8-10)$. (h) Fasted gastrointestinal (Gl) motor activity in tumor-bearing rats on day 5 . Ghrelin increased the frequency of phase III-like contractions $(\mathbf{\Lambda})$ in the duodenum $(n=8) .{ }^{*} P<0.05 ;{ }^{* \star} P<0.01$. AgRP: agouti-related peptide; CART: cocaine- and amphetamine-regulated transcript; MCH: melanin-concentrating hormone; OXT: oxytocin; UCN, urocortin. 


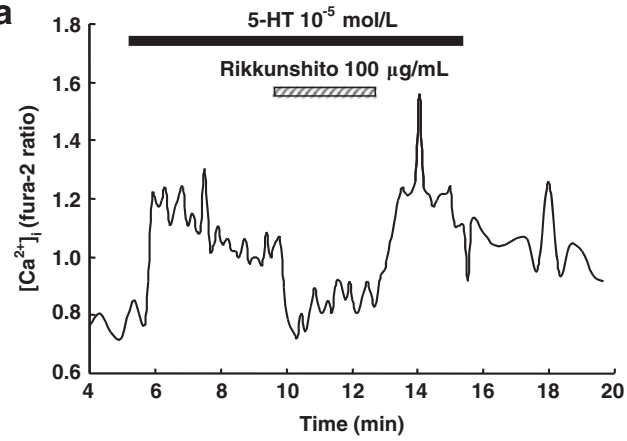

b

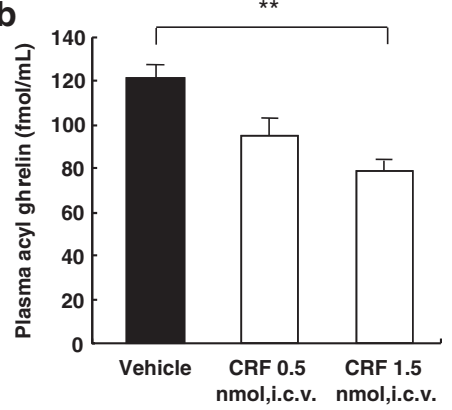

C

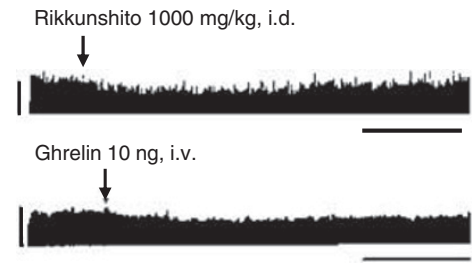

Vertical bars: 100 impulses $/ 5 \mathrm{~s}$, Horizontal bars: $30 \mathrm{~min}$
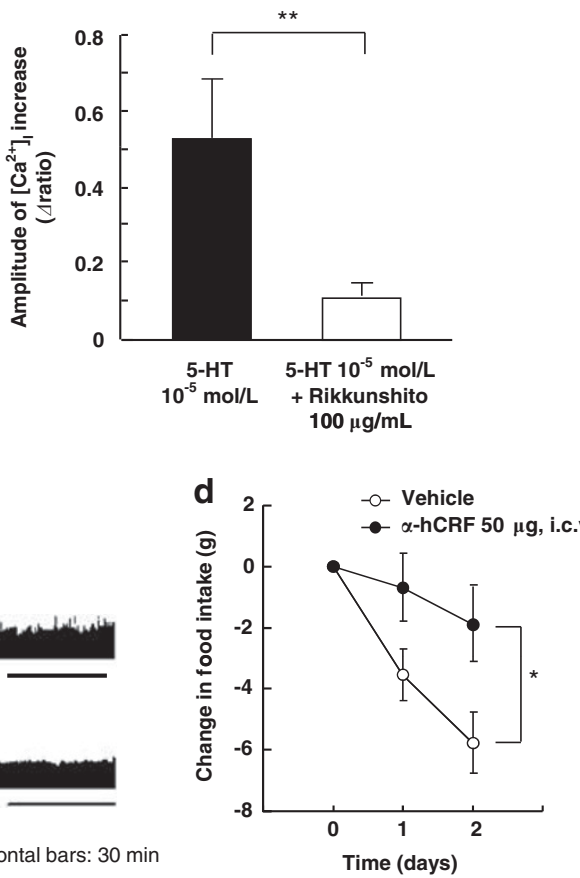
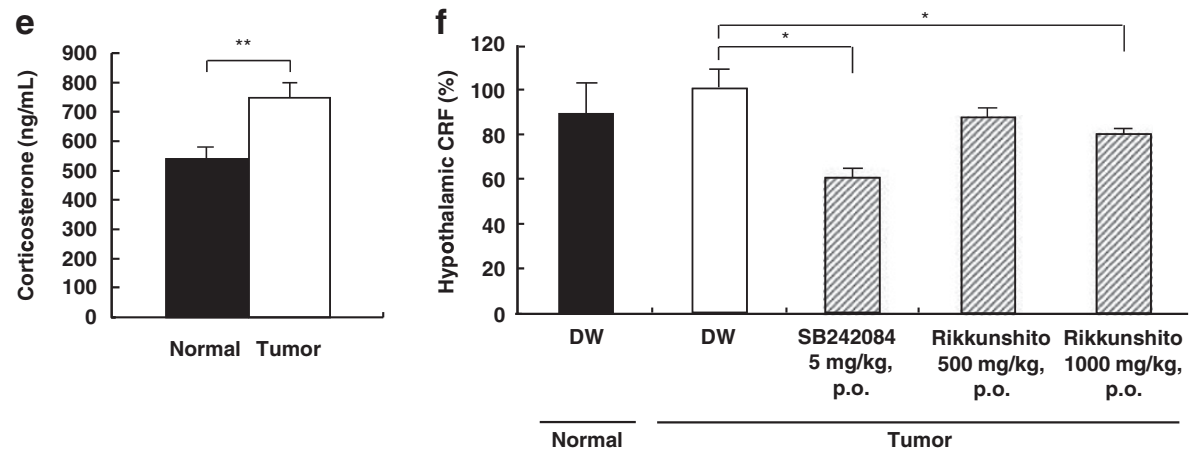

g
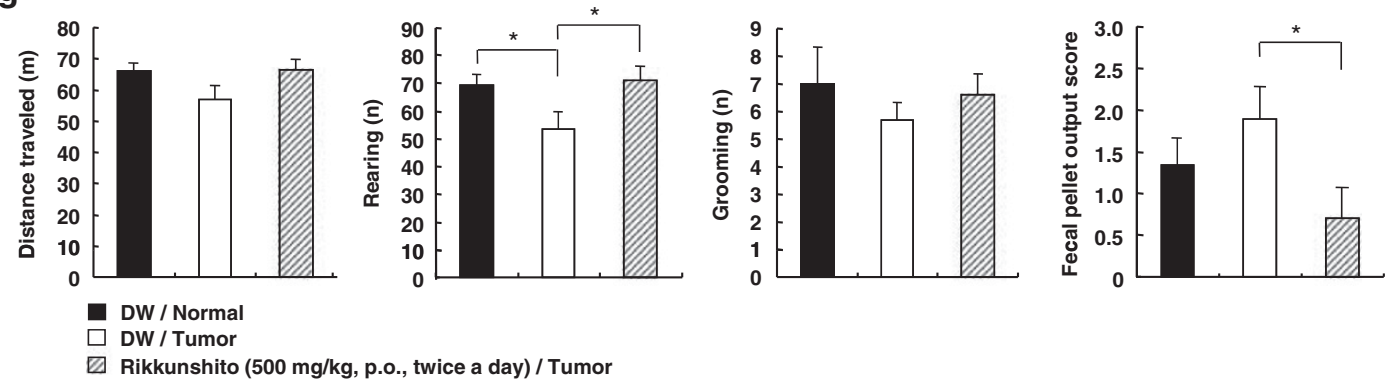

Figure 2 Involvement of corticotropin-releasing factor (CRF) in cancer anorexia. (a) The serotonin (5-HT)-induced cytosolic $\mathrm{Ca}^{2+}$ concentration ([Ca $\left.\left.{ }^{2+}\right]_{\mathrm{i}}\right)$ increase was suppressed by rikkunshito in single CRF neurons isolated from the paraventricular nucleus (PVN). (b) Inhibitory effect of CRF on plasma acyl ghrelin concentrations in fasted rats $(n=8-9)$. (c) Inhibitory effects of ghrelin and rikkunshito on the efferent activity of the adrenal sympathetic nerve in rats. (d) Food intake was increased in tumor-bearing rats by daily administration of the CRF receptor antagonist $\alpha$-helical CRF $(n=9-10)$. (e) Plasma corticosterone concentration of tumor-bearing rats $(n=12)$. (f) Hypothalamic CRF levels in tumor-bearing rats were decreased by SB242084 or rikkunshito $(n=10)$. (g) Daily administration of rikkunshito in tumor-bearing rats improved rearing and decreased fecal pellet output score in an open-field test $(n=9-10)$. ${ }^{*} P<0.05$; ${ }^{* *} P<0.01$. DW: distilled water.

rikkunshito $\left(1000 \mathrm{mg} \mathrm{kg}^{-1}\right)$. Gastric vagotomy eliminated the stimulatory effect of ghrelin (10 ng, intravenous) on the efferent activities of the gastric vagus nerve, but did not influence the effect of rikkunshito $\left(1000 \mathrm{mg} \mathrm{kg}^{-1}\right.$, intraduodenal) or a 100 -fold higher dose of ghrelin (1000 ng, intravenous) (Supplementary Figure S3). 
a

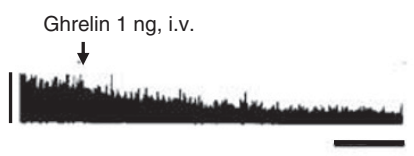

Rikkunshito 1000 mg/kg, i.d.

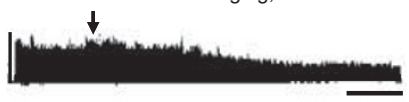

Vertical bars: 100 impulses $/ 5 \mathrm{~s}$, Horizontal bars: 30 min b

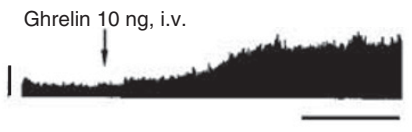

Rikkunshito 1000 mg/kg, i.d.

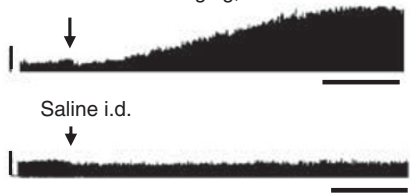

Vertical bars: 100 impulses/5s, Horizontal bars: $30 \mathrm{~min}$
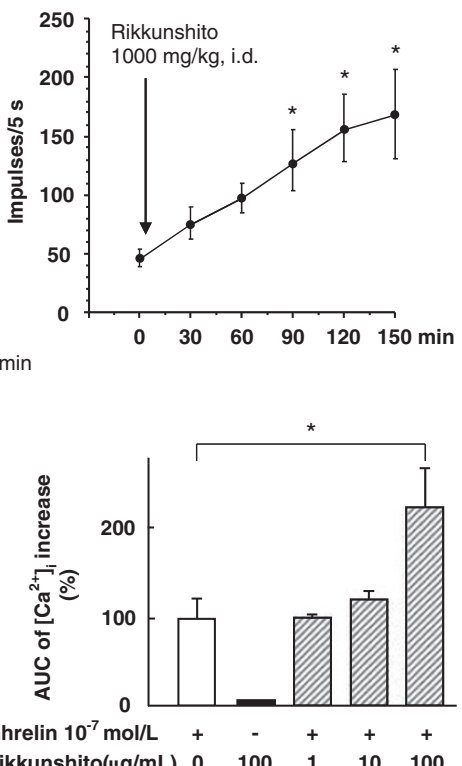

$\begin{array}{llcccc}\text { Ghrelin } 10^{-7} \mathrm{~mol} / \mathrm{L} & + & - & + & + & + \\ \text { Rikkunshito }(\mu \mathrm{g} / \mathrm{mL}) & 0 & 100 & 1 & 10 & 100\end{array}$

e

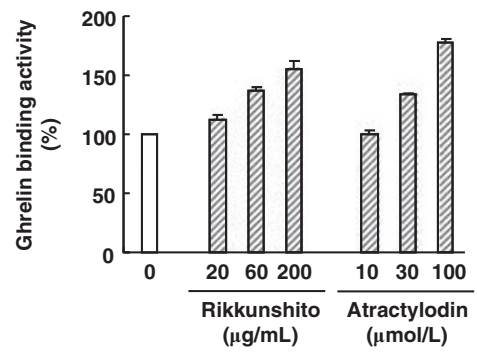

g

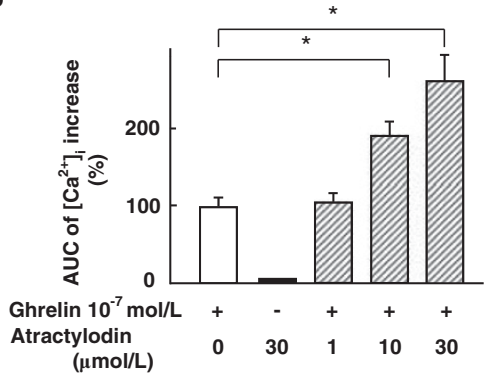

i h

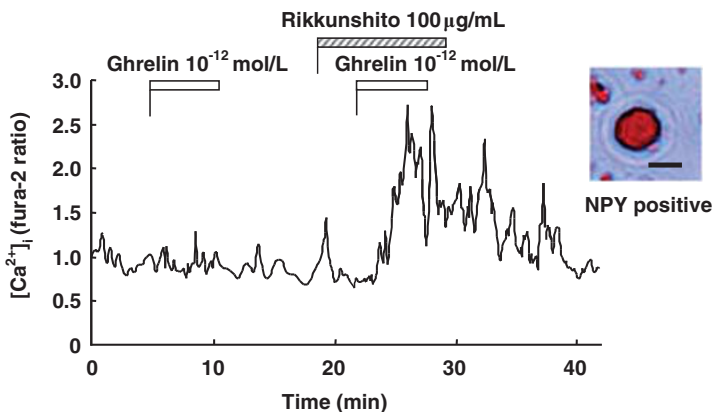

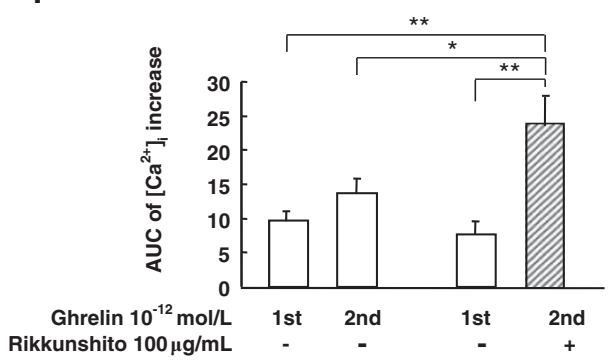

Figure 3 Ghrelin signaling and rikkunshito. (a, b) Effects of ghrelin and rikkunshito on the afferent (a) and efferent (b, $n=6)$ activities of the gastric vagus nerve in rats. (c, d) Changes in $\mathrm{F} / \mathrm{F}_{0}$ fluorescence evoked by ghrelin in ghrelin receptor (GHS-R)-expressing cells. Representative $\mathrm{Ca}^{2+}$-imaging figures were taken as indicated by the arrowheads in the figures (a-d) and the intensities within the area of a cell (red line). $\left[\mathrm{Ca}^{2+}\right]_{\text {i }}$ increase in the area under the curve (AUC) was evaluated $(n=6-9)$. (e) Effect of rikkunshito and atractylodin on ghrelin/GHS-R binding activity. (f, g) Atractylodin enhanced the ghrelin-induced $\left[\mathrm{Ca}^{2+}\right]_{\mathrm{i}}$ increase in GHS-R-expressing cells $(n=8-12)$. (h, i) Ghrelin $\left(10^{-12}\right.$ mol I $\left.^{-1}\right)$ increased the $\left[\mathrm{Ca}^{2+}\right]_{\mathrm{i}}$ in single neuropeptide $\mathrm{Y}$ (NPY) neurons isolated from the arcuate nucleus (ARC). The increase in $\mathrm{AUC}$ of the [Ca $\left.{ }^{2+}\right]_{\mathrm{i}}$ in response to secondary ghrelin with rikkunshito $(n=22)$ was significantly greater than the response to primary or secondary ghrelin without rikkunshito $(n=32)$. $P<0.05$; ${ }^{\star \star} P<0.01$. 
Ghrelin is predominantly produced in gastric X/A-like cells. Rikkunshito elevated the gene expression of gastric ghrelin and hypothalamic NPY genes in rats (Supplementary Figure S4a). Rikkunshito did not acutely stimulate ghrelin secretion from minced stomach tissues (data not shown), but normalized the fenfluramine-induced decrease in ghrelin secretion mediated by the $5-\mathrm{HT} 2 \mathrm{cR}$ in the brain (Supplementary Figure $\mathrm{S} 4 \mathrm{~b})$, suggesting that this is the centrally predominant site of action.

Ghrelin $\left(10^{-7} \mathrm{moll}^{-1}\right)$ elicited an increase in $\left[\mathrm{Ca}^{2+}\right]_{\mathrm{i}}$ of GHS-R-expressing COS cells. Rikkunshito had no effect on the $\left[\mathrm{Ca}^{2+}\right]_{i}$ in these cells. However, the ghrelin-induced $\left[\mathrm{Ca}^{2+}\right]_{i}$ increase was enhanced by a 2-min pretreatment with rikkunshito in a concentration-dependent manner, and $100 \mu \mathrm{g} \mathrm{ml}^{-1}$ of rikkunshito significantly enhanced the duration of the $\left[\mathrm{Ca}^{2+}\right]_{i}$ increase induced by ghrelin (Figure $3 \mathrm{c}$ and $\mathrm{d}$ ).

Rikkunshito enhanced the binding activity of $\left[{ }^{125} \mathrm{I}\right]$ ghrelin to the GHS-R (Figure 3e). We screened the 43 compounds $\left(100 \mu \mathrm{mol} \mathrm{I}^{-1}\right)$ contained in rikkunshito. Two of these compounds, atractylodin and atractylodinol, showed a marked increase in ghrelin/GHS-R binding activity. Atractylodin also sustained the ghrelin-induced $\left[\mathrm{Ca}^{2+}\right]_{i}$ increase in GHS-Rexpressing cells (Figure $3 f$ and $g$ ).

Ghrelin increases the $\left[\mathrm{Ca}^{2+}\right]_{i}$ in the NPY neurons of the hypothalamic ARC, ${ }^{18}$ and this effect is linked to stimulation of feeding. ${ }^{19}$ Ghrelin, at a submaximal concentration of $10^{-12} \mathrm{moll}^{-1}$, increased $\left[\mathrm{Ca}^{2+}\right]_{\mathrm{i}}$ levels in acutely isolated fura-2-loaded rat ARC neurons, which were subsequently shown to be NPY neurons by immunocytochemistry. Pretreatment with rikkunshito enhanced the ghrelin-induced increase in $\left[\mathrm{Ca}^{2+}\right]_{\mathrm{i}}$ compared with first ghrelin or second ghrelin administration without rikkunshito (Figure $3 \mathrm{~h}$ and i). These data indicate that rikkunshito potentiates the action of ghrelin to increase the $\left[\mathrm{Ca}^{2+}\right]_{i}$ in NPY neurons in the ARC.

Survival. Daily administration of (D-Lys3)-GHRP-6 $\left(4 \mu \mathrm{mol} \mathrm{kg}^{-1}\right.$, i.p.; Figure $\left.4 \mathrm{a}\right)$ decreased median survival in $\mathrm{AH}-130$ tumor-bearing rats, demonstrating the importance of ghrelin signaling in cancer anorexia-cachexia. In contrast, median survival in $\mathrm{AH}-130$ tumor-bearing rats was significantly increased by the daily administration of rikkunshito (250 and $500 \mathrm{mg} \mathrm{kg}^{-1}$, per os; Figure 4b) and atractylodin ( $1 \mathrm{mg} \mathrm{kg}^{-1}$, per os; Figure 4c), but not SB242084 $\left(5 \mathrm{mg} \mathrm{kg}^{-1}\right.$, per os) or ghrelin ( $3 \mathrm{nmol}$, i.p.) (Supplementary Figure S5). Rikkunshito also exhibited a positive effect on survival in CT-26 colon carcinoma-bearing mice (Supplementary Figure S6). Survival in tumor-bearing rats was also increased by administration of cisplatin (CDDP; $1 \mathrm{mg} \mathrm{kg}^{-1}$, i.p.), and 6 of 27 rats survived until the end of the experimental period. Administration of rikkunshito further prolonged survival in CDDP-treated tumor-bearing rats (Figure 4d).

The beneficial effect of rikkunshito on survival was also demonstrated in human patients. Pancreatic cancer patients with ascites received gemcitabine or gemcitabine plus rikkunshito. There was no significant difference between the two groups in baseline data with respect to stage and age. Median survival of pancreatic cancer patients with ascites who were treated with gemcitabine was significantly prolonged by administration of rikkunshito (Figure $4 \mathrm{e}$ ).

\section{Discussion}

Weight loss is a potent stimulus of food intake in healthy humans and animals, and ghrelin secretion increases under conditions of negative energy balance such as starvation. Increased concentrations of acyl ghrelin have been found in patients ${ }^{20,21}$ and mice $^{22}$ with various cancer diagnoses and staging. These findings imply that the persistence of anorexia in cancer patients is due to a failure of the adaptive feeding response by ghrelin, which is robust in normal animals and subjects. ${ }^{23-25}$ We found that plasma acyl ghrelin concentrations in tumor-bearing rats were higher than that in free-fed normal rats, but lower than that in pair-fed normal rats, and had an inverse relationship with plasma leptin concentrations. These results indicate that changes in ghrelin and leptin secretion in pair-fed animals represent a compensatory mechanism in a persistent catabolic state and that these responses are attenuated in tumor-bearing rats. The hypothermia in tumor-bearing rats may be due to a state of negative energy balance or a decrease in the threshold for the activation of thermogenesis, which is involved in starvationinduced hypothermia. ${ }^{26}$ Interleukin- $1 \beta^{17}$ and leptin ${ }^{27}$ decrease the expression of ghrelin mRNA in the stomach, whereas interleukin- 6 produced in various cells, including adipocytes, regulates leptin production. ${ }^{28}$ These findings suggest that cytokines have an important role in energy balance through the persistent activation of the leptin system and the inhibition of the ghrelin-NPY/agouti-related peptide orexigenic network in tumor-bearing rats. In addition to NPY and agouti-related peptide, the level of POMC mRNA was also decreased in the hypothalamus of the tumor-bearing rats. Synaptic input organization and mRNA expression of POMC neuron have been shown to be increased in adrenalectomized animals and restored by corticosterone replacement. ${ }^{29}$ Thus, activity of hypothalamic POMC neuron may be affected by changes in circulating levels of corticosterone and a state of negative energy balance.

Peripheral ghrelin administration stimulates food intake in melanoma cell-bearing mice and cancer patients ${ }^{30}$ in the short term as well as in lean, healthy men and women. ${ }^{31}$ In this study, we found similar therapeutic effects of ghrelin on anorexia and Gl dysmotility in cachectic animal models, suggesting that high plasma concentrations of ghrelin may overcome resistance to the appetite-stimulating effects of the endogenous peptide in the short term. Rikkunshito, which mimics these ghrelin effects, effectively improved food intake and GI motor activities in this study. Rikkunshito is a traditional herbal medicine used to treat GI tract disorders such as functional dyspepsia ${ }^{32-36}$ and gastroesophageal reflux. ${ }^{37}$ Oral administration of rikkunshito increases plasma acyl ghrelin levels in humans, mice, ${ }^{38}$ rats $^{14,15}$ and dogs (data not shown). Rikkunshito stimulates ghrelin secretion through $5-\mathrm{HT} 2 \mathrm{~b} / 2 \mathrm{c}$ receptor antagonism, and its active flavonoid ingredients such as hesperidin that antagonize $5-\mathrm{HT} 2 \mathrm{~b} / 2 \mathrm{c}$ receptor binding have been identified. ${ }^{14}$ In addition, rikkunshito and 5-HT2cR antagonist suppress cisplatininduced anorexia by inhibiting reduction of GHS-R1a gene expression in the hypothalamus. ${ }^{39}$

The central 5-HT system has been implicated in the processes of meal satiation and satiety. 5-HT reuptake 


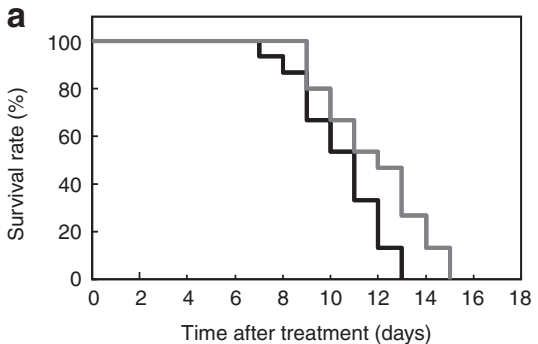

Median survival

Saline (i.p.) : 12 days $(n=15)$

(D-Lys3)-GHRP-6 $(4 \mu \mathrm{mol} / \mathrm{kg}$, i.p.) : 11 days $(n=15)$

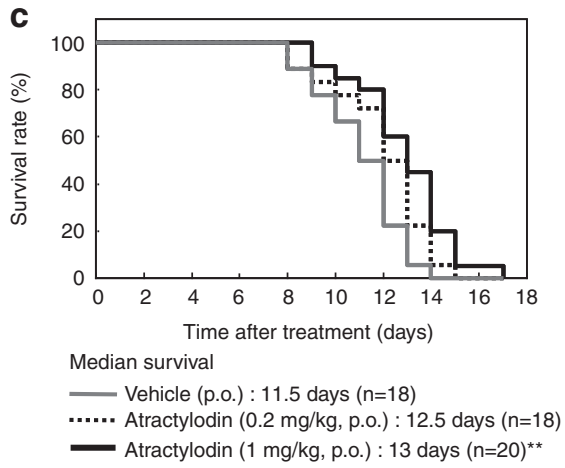

b

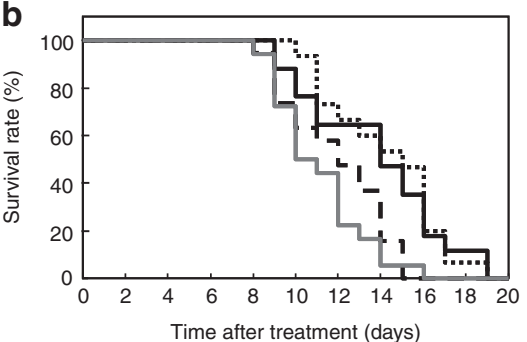

Median surviva

DW (p.o.) : 10.5 days $(n=18)$

- Rikkunshito $(125 \mathrm{mg} / \mathrm{kg}$, p.o.) : 12 days $(\mathrm{n}=19)$

...... Rikkunshito $(250 \mathrm{mg} / \mathrm{kg}$, p.o.) : 15 days $(n=15)$ **

Rikkunshito $(500 \mathrm{mg} / \mathrm{kg}$, p.o.) : 14 days $(n=17)$ **

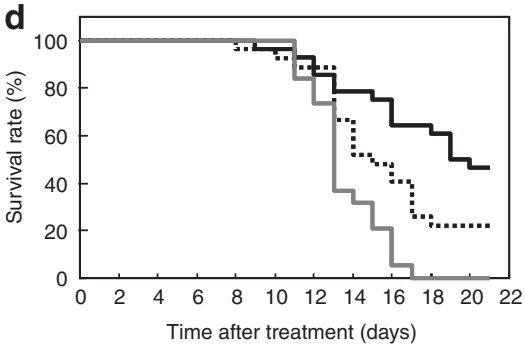

Median survival

- Saline + DW (p.o.) : 13 days $(n=19)$

$\cdots . . . \operatorname{CDDP}(1 \mathrm{mg} / \mathrm{kg}$, i.p. $)+$ DW (p.o.) : 15 days $(\mathrm{n}=27)$ **

CDDP (1 mg/kg, i.p.) + Rikkunshito $(500 \mathrm{mg} / \mathrm{kg}$, p.o.) 19.5 days $(\mathrm{n}=28)^{* *}, \#$

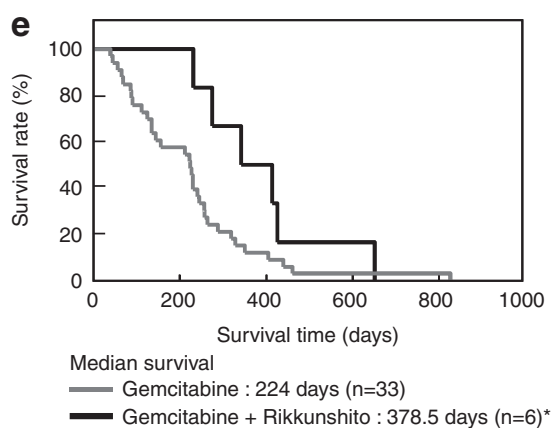

Figure 4 Survival of tumor-bearing rats and patients with pancreatic cancer. (a) Administration of (D-Lys3)-GHRP-6 decreased the median survival of tumor-bearing rats. (b, c) Administration of rikkunshito and atractylodin increased the median survival of tumor-bearing rats. (d) Survival of tumor-bearing rats was increased by intraperitoneal (i.p.) administration of cisplatin (CDDP) and further prolonged by co-administration of rikkunshito. (e) Rikkunshito prolonged the median survival of pancreatic cancer patients with ascites who were treated with gemcitabine. ${ }^{*} P<0.05 ;{ }^{\star *} P<0.01$ vs control group; ${ }^{\sharp} P<0.05$ vs CDDP + DW. DW: distilled water.

inhibitors such as fenfluramine and 5-HT2cR agonists attenuate food intake and weight gain in rodents and humans, ${ }^{40-42}$ with the involvement of potentiated MC signaling and decreased ghrelin secretion. 5-HT also inhibits NPY/ agouti-related peptide neurons by activating the $5-\mathrm{HT} 1 \mathrm{bR}$, leading to decreased orexigenic signaling and an inhibitory drive onto POMC cells. However, our previous study suggested that the $5-\mathrm{HT} 2 \mathrm{cR}$ has a major role in the regulation of physiological fasted and fed motor activities in addition to feeding through changes in endogenous ghrelin. ${ }^{15}$ In this study, we found that the decreases in food intake and Gl motor activities in tumor-bearing rats were recovered after administration of either a 5-HT2cR antagonist or ghrelin. The $5-\mathrm{HT}$ concentration in the hypothalamus is increased in humans and animals with cancer; ${ }^{43,44}$ in addition, NPY and dopamine concentrations decrease simultaneously, while 5$\mathrm{HT}$ concentration increases in the PVN at the onset of anorexia in tumor-bearing rats. ${ }^{45}$ These findings suggest that $5-\mathrm{HT} 2 \mathrm{cR}$ activation in tumor-bearing rats induces anorexia in part via decreased ghrelin secretion.

We have previously shown that a central $5-\mathrm{HT} 2 \mathrm{cR}$ pathway regulates ghrelin secretion without downstream activation of melanocortin $3 / 4$ receptors. ${ }^{15}$ The $5-\mathrm{HT} 2 \mathrm{cR}$ is expressed in many brain regions and its expression is restricted to the central nervous system. ${ }^{46}$ Dual-neurohistochemical labeling has revealed that approximately one-half of PVN CRFcontaining neurons co-express 5 -HT2cR mRNA. ${ }^{47}$ In this study, we found that $5-\mathrm{HT}$ activated single CRF neurons isolated from the PVN, and the activities of the CRF neurons were blocked by simultaneous administration of rikkunshito. 
Moreover, intracerebroventricular administration of CRF decreased plasma acyl ghrelin in fasted rats. These findings suggest that CRF neurons are involved in 5-HT-regulated ghrelin secretion. Hypothalamic 5-HT and CRF activities are stimulated by proinflammatory cytokines in the circulation and the hypothalamus. ${ }^{13,48}$ Here we demonstrate that a CRF receptor antagonist improved cancer anorexia-cachexia, and that administration of the $5-\mathrm{HT} 2 \mathrm{cR}$ antagonist or rikkunshito reduced hypothalamic CRF levels and anxiety-related behaviors in tumor-bearing rats. The improvement in anxiety by rikkunshito may lead to a higher quality of life in cancer patients. Some studies suggest that ghrelin induce anxiogenesis, whereas others suggest that the elevated ghrelin helps animals cope with stress by producing anxiolytic-like response. ${ }^{49}$ Future studies are needed to sort out the effect of ghrelin on anxiety-like behavior as in the case for NPY ${ }^{50}$ Importantly, our findings demonstrate that a hypothalamic 5HT-CRF receptor pathway that regulates ghrelin secretion has a major role in cancer anorexia-cachexia.

The GHS-R is reportedly expressed in vagal afferent neurons, and the gastric vagus nerve system is involved in the effect of ghrelin on food intake and Gl motor activities. ${ }^{51,52}$ We demonstrated that ghrelin decreased the afferent activity of the gastric vagus nerve. Gastric ghrelin signaling via vagal afferents stimulated the efferent activities of both the gastric and the celiac branches of the vagus nerve and suppressed the activity of the sympathetic nerve. Peripheral administration of a higher dose of ghrelin increased the discharge rate of the vagal efferent nerve, probably in part through the GHS-R in the ARC of the hypothalamus. We also showed that rikkunshito activated the efferent vagus nerve, which may be mediated by both the vagal afferent nerve and the direct central action (Figure 5). In addition, we found that ghrelininduced cellular signaling in GHS-R-expressing cells was enhanced by pretreatment with rikkunshito and its active components, such as atractylodin, which stimulate ghrelin/ GHS-R binding activity. Similar potentiating effects of rikkunshito were observed in rat ARC NPY neurons. These findings suggest that the physiological functions of endogenous ghrelin are enhanced by the dual actions of rikkunshito, which involve the stimulation of ghrelin secretion and the activation of GHS-R activity, possibly due to allosteric changes in the receptor. This potentiation of the ghrelin effect by rikkunshito on NPY neurons could be orexigenic because the activity of ghrelin-responsive NPY neurons is coupled to feeding. ${ }^{19,53}$

Our study indicated the adverse effect of (D-Lys3)-GHRP-6 on survival in tumor-bearing rats, suggesting that the potentiation of ghrelin signaling is critical to the attenuation of anorexia-cachexia and the prolongation of survival in subjects with cancer. Rikkunshito and its active component, atractylodin, prolonged survival in these animals, and this effect was enhanced by the concomitant administration of CDDP. Cancer patients receiving chemotherapy or radiation therapy may experience nausea, vomiting, taste changes, stomatitis and diarrhea, which could contribute to weight loss and decreased survival. Therefore, cancer anorexiacachexia syndrome is a major obstacle in cancer chemotherapy. ${ }^{6}$ Rikkunshito was recently reported to suppress decreases in plasma acyl ghrelin levels and CDDP-induced
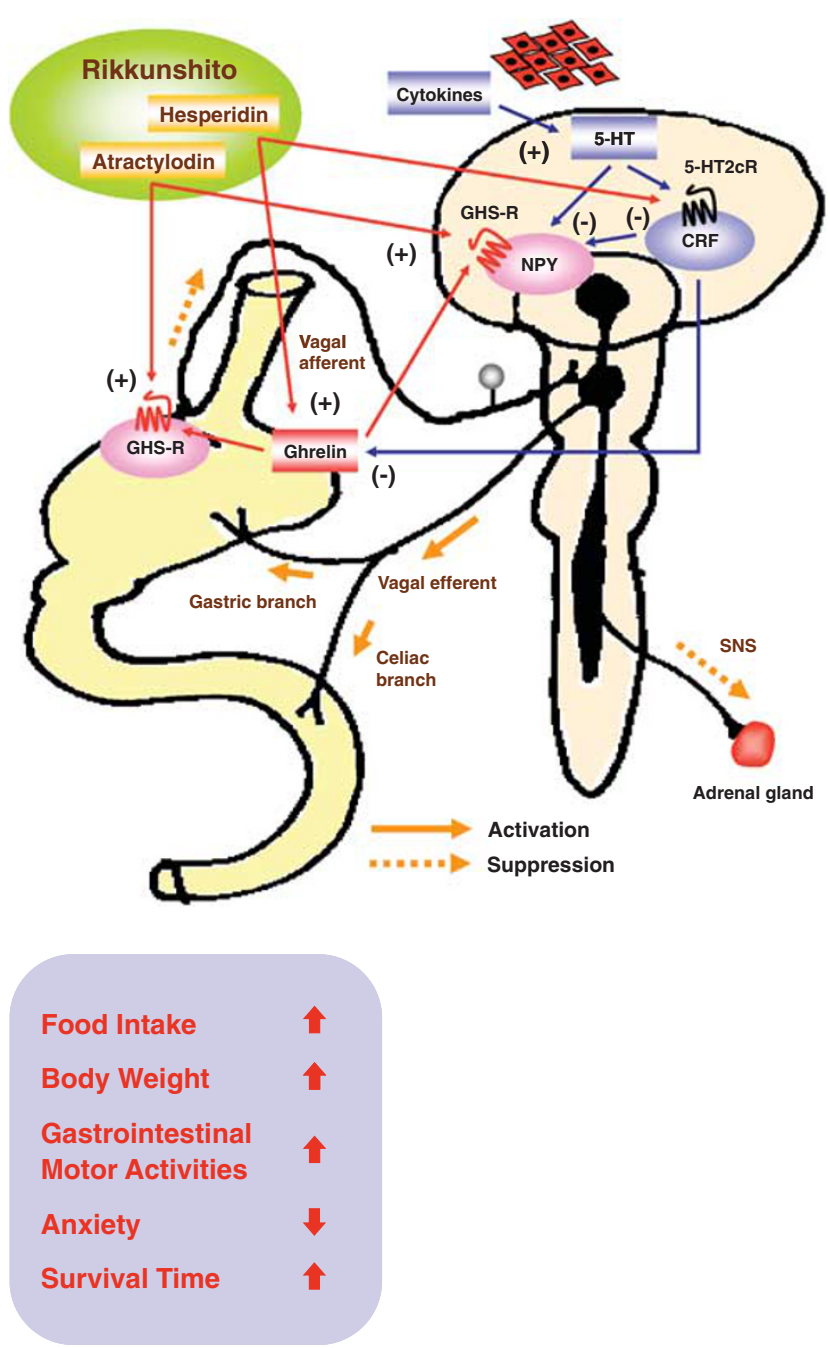

Figure 5 Ghrelin signaling and cancer anorexia-cachexia. Hypothalamic corticotropin-releasing factor (CRF) neurons are activated by cytokines through serotonin $(5-\mathrm{HT})$ and the $5-\mathrm{HT} 2 \mathrm{c}$ receptor $(5-\mathrm{HT} 2 \mathrm{cR})$, which shows functional divergence. ${ }^{15}$ Our data demonstrate the existence of a novel 5 -HT-CRF neuronal pathway that inhibits ghrelin secretion and has a pathogenetic role in cancer anorexia-cachexia syndrome. The traditional herbal medicine rikkunshito improves anorexia, weight loss, gastrointestinal (GI) dysmotility, anxiety-related behavior and survival. Rikkunshito and its active component hesperidin stimulate ghrelin secretion from stomach by interrupting this 5 -HT-CRF pathway via $5-\mathrm{HT} 2 \mathrm{CR}$ antagonism. Another active component atractylodin potentiates the action of ghrelin by presumably allosterically sensitizing the GHS-R on the vagal afferent terminals of stomach or neuropeptide $Y$ (NPY) neurons of the hypothalamic arcuate nucleus (ARC). The 5-HT2CR antagonist improved anorexia-cachexia in the short term, but failed to improve survival. Thus, both the release of ghrelin and the potentiation of ghrelin/GHS-R signaling are important for mitigating ghrelin insufficiency and resistance, which are characteristics of cancer anorexia-cachexia syndrome.

anorexia. ${ }^{14}$ Our use of rikkunshito in tumor-bearing rats was effective not only against anorexia-cachexia, but also for promoting survival, particularly in combination with chemotherapy. Daily administration of a 5-HT2c receptor antagonist failed to prolong survival, suggesting that a sensitizing effect on the GHS-R may be essential for ameliorating ghrelin resistance in anorexia-cachexia in the long term. Pancreatic cancer patients generally respond poorly to chemotherapy, 
resulting in a higher frequency of anorexia-cachexia. We found that the median survival of pancreatic cancer patients treated with gemcitabine was prolonged by the addition of rikkunshito, particularly for those with ascites. These results suggest that rikkunshito may be useful in clinical practice for cachectic cancer patients via its dual action on ghrelin secretion and receptor sensitization.

In conclusion, our study demonstrates that cancer anorexiacachexia is mediated by decreased ghrelin/GHS-R signaling as a result of excessive hypothalamic interactions of 5-HT and CRF through the 5-HT2cR. Decreased ghrelin signaling is an important integrated mechanism linking anorexia, body weight loss, Gl dysmotility, anxiety-related behavior and decreased survival. Potentiation of ghrelin signaling introduces a novel approach for the treatment of cancer anorexia-cachexia, which is characterized by ghrelin insufficiency and resistance.

\section{Conflict of interest}

Al has received grant support from Tsumura \& Co. The remaining authors have nothing to declare.

Acknowledgements. This work was supported by Grants-in-Aid for Scientific Research from the Ministry of Education, Culture, Sports, Science and Technology, and the Ministry of Health Labour and Welfare of Japan.

Author contributions. $\mathrm{AA}$ and $\mathrm{Al}$ supervised the project and designed experiments. NF, AN, TS, TH and YK performed the experiments. YU, KM, To Y, YM and US performed the cellular analysis. TaY analyzed the survival data in cancer patients. NF, AA and Al wrote the paper. All authors critically discussed the results and the manuscript as a whole.

Ethics statement. All experimental procedures were performed according to the 'Guidelines for the Care and Use of Laboratory Animals' approved by the Institutional Review Boards of the National Cancer Center Research Institute, the Jichi Medical University and Tsumura. Humane end points were chosen to minimize or terminate pain or distress in the animals via euthanasia, rather than waiting for death as an end point. All research involving human participants was approved by the Institutional Review Board of Chiba Cancer Center. The study did not require patient consent, as it was a retrospective analysis. However, participants gave written or verbal consent. Data were anonymized and no patient identifying information was included. All clinical investigation was conducted according to the principles expressed in the Declaration of Helsinki.

1. Tisdale MJ. Biology of cachexia. J Natl Cancer Inst 1997; 89: 1763-1773.

2. Mantovani G, Maccio A, Massa E, Madeddu C. Managing cancer-related anorexia/ cachexia. Drugs 2001; 61: 499-514.

3. Inui A. Eating behavior in anorexia nervosa-an excess of both orexigenic and anorexigenic signalling? Mol Psychiatry 2001; 6: 620-624.

4. Perboni S, Inui A. Anorexia in cancer: role of feeding-regulatory peptides. Philos Trans $R$ Soc Lond Ser B 2006; 361: 1281-1289.

5. Morley JE, Farr SA. Cachexia and neuropeptide Y. Nutrition 2008; 24: 815-819.

6. Inui A. Cancer anorexia-cachexia syndrome: current issues in research and management CA Cancer J Clin 2002; 52: 72-91.

7. Jatoi A, Yamashita J, Slotan JA, Novotny PJ, Windschitl HE, Loprinzi CL. Does megastrol acetate down-regulate interleukin-6 in patients with cancer-associated anorexia and weigh loss? A North Central Cancer Treatment Group Investigation. Support Care Cancer 2002; 10: $71-75$.

8. Nelson KA. The cancer anorexia-cachexia syndrome. Semin Oncol 2000; 27 64-68

9. North CS, Hong BA, Alpers DH. Relationship of functional gastrointestinal disorders and psychiatric disorders: implications for treatment. World J Gastroenterol 2007; 13: 2020-2027.

10. Levy RL, Olden KW, Naliboff BD, Bradley LA, Francisconi C, Drossman DA et al. Psychosocial aspects of the functional gastrointestinal disorders. Gastroenterology 2006; 130: $1447-1458$.
11. Smagin GN, Dunn AJ. The role of CRF receptor subtypes in stress-induced behavioural responses. Eur J Pharmacol 2000; 405: 199-206.

12. Inui A. Feeding and body-weight regulation by hypothalamic neuropeptides-mediation of the actions of leptin. Trends Neurosci 1999; 22: 62-67.

13. Chen CY, Fujimiya M, Laviano A, Chang FY, Lin HC, Lee SD. Modulation of ingestive behavior and gastrointestinal motility by ghrelin in diabetic animals and humans. $J$ Chin Med Assoc 2010; 73: 225-229.

14. Takeda H, Sadakane $C$, Hattori T, Katsurada T, Ohkawara T, Nagai K et al. Rikkunshito, an herbal medicine, suppresses cisplatin-induced anorexia in rats via 5-HT2 receptor antagonism. Gastroenterology 2008; 134: 2004-2013.

15. Fujitsuka N, Asakawa A, Hayashi M, Sameshima M, Amitani H, Kojima S et al. Selective serotonin reuptake inhibitors modify physiological gastrointestinal motor activities via 5HT2c receptor and acyl ghrelin. Biol Psychiatry 2009; 65: 748-759.

16. Sutton RE, Koob GF, Le Moal M, Rivier J, Vale W. Corticotropin releasing factor produces behavioural activation in rats. Nature 1982; 297: 331-333.

17. Asakawa A, Inui A, Kaga T, Yuzuriha H, Nagata T, Ueno N et al. Ghrelin is an appetitestimulatory signal from stomach with structural resemblance to motilin. Gastroenterology 2001; 120: 337-345.

18. Kohno D, Gao HZ, Muroya S, Kikuyama S, Yada T. Ghrelin directly interacts with NPYcontaining neurons in the rat arcuate nucleus: $\mathrm{Ca}^{2+}$ signaling via protein kinase $\mathrm{A}$ - and $\mathrm{N}$ type channel-dependent mechanisms and cross-talk with leptin and orexin. Diabetes 2003; 52: $948-956$

19. Kohno D, Nakata M, Maekawa F, Fujiwara K, Maejima $Y$, Kuramochi M et al. Leptin suppresses ghrelin-induced activation of neuropeptide $Y$ neurons in the arcuate nucleus via phosphatidylinositol 3-kinase- and phosphodiesterase 3-mediated pathway. Endocrinology 2007; 148: 2251-2263.

20. Shimizu $\mathrm{Y}$, Nagaya N, Isobe T, Imazu M, Okumura H, Hosoda $\mathrm{H}$ et al. Increased plasma ghrelin level in lung cancer cachexia. Clin Cancer Res 2003; 9: 774-778.

21. Garcia JM, Garcia-Touza M, Hijazi RA, Taffet G, Epner D, Mann D et al. Active ghrelin levels and active to total ghrelin ratio in cancer-induced cachexia. J Clin Endocrinol Metab 2005; 90: 2920-2926.

22. Hanada T, Toshinai K, Date $Y$, Kajimura N, Tsukada T, Hayashi $Y$ et al. Upregulation of ghrelin expression in cachectic nude mice bearing human melanoma cells. Metabolism 2004; 53: 84-88.

23. Flier JS. Clinical review 94: What's in a name? In search of leptin's physiologic role. J Clin Endocrinol Metab 1998; 83: 1407-1413.

24. Schwartz MW, Dallman MF, Woods SC. Hypothalamic response to starvation: implications for the study of wasting disorders. Am J Physiol 1995; 269: 949-957.

25. Schwartz MW, Seeley RJ. Seminars in medicine of the Beth Israel Deaconess Medical Center. Neuroendocrine responses to starvation and weight loss. N Engl J Med 1997; 336: 1802-1811.

26. Sakurada S, Shido O, Sugimoto N, Hiratsuka Y, Yoda T, Kanosue K. Autonomic and behavioural thermoregulation in starved rats. J Physiol 2000; 526: 417-424.

27. Zhao Z, Sakata I, Okubo Y, Koike K, Kangawa K, Sakai T. Gastric leptin, but not estrogen and somatostatin, contributes to the elevation of ghrelin mRNA expression level in fasted rats. J Endocrinol 2008; 196: 529-538

28. Turrin NP, Ilyin SE, Gayle DA, Plata-Salaman CR, Ramos EJ, Laviano A et al. Interleukin1 beta system in anorectic catabolic tumor-bearing rats. Curr Opin Clin Metab Care 2004; 7: 419-426.

29. Gyengesi E, Gyengesi E, Liu ZW, D'Agostino G, Gan G, Horvath TL et al. Corticosterone regulates synaptic input organization of POMC and NPY/AgRP neurons in adult mice. Endocrinology 2010; 151: 5395-5402.

30. Neary NM, Small CJ, Wren AM, Lee JL, Druce MR, Palmieri C et al. Ghrelin increases energy intake in cancer patients with impaired appetite: acute, randomized, placebocontrolled trial. J Clin Endocrinol Metab 2004; 89: 2832-2836.

31. Laferrere B, Abraham C, Russell CD, Bowers CY. Growth hormone releasing peptide-2 (GHRP-2), like ghrelin, increases food intake in healthy men. J Clin Endocrinol Metab 2005; 90: $611-614$

32. Kusunoki $\mathrm{H}$, Haruma K, Hata J, Ishii M, Kamada T, Yamashita $\mathrm{N}$ et al. Efficacy of rikkunshito, a traditional Japanese medicine (Kampo), in treating functional dyspepsia. Intern Med 2010; 49: 2195-2202.

33. Takahashi T, Endo S, Nakajima K, Souma Y, Nishida T. Effect of rikkunshito, a Chinese herbal medicine, on stasis in patients after pylorus-preserving gastrectomy. World J Surg 2009; 33: 296-302.

34. Oyachi N, Takano K, Hasuda N, Arai H, Koshizuka K, Matsumoto M. Effects of Rikkunshito on infantile hypertrophic pyloric stenosis, refractory to atropine. Pediatr Int 2008; 50: 581-583.

35. Oka T, Tamagawa Y, Hayashida S, Kaneda Y, Kodama N, Tsuji S. Rikkunshito attenuates adverse gastrointestinal symptoms induced by fluvoxamine. Biopsychosoc Med 2007; 1: 21

36. Yagi M, Homma S, Kubota M, linuma Y, Kanada S, Kinoshita $Y$ et al. The herbal medicine Rikkunshito stimulates and coordinates the gastric myoelectric activity in post-operative dyspeptic children after gastrointestinal surgery. Pediatr Surg Int 2004; 19: 760-765.

37. Kawahara H, Okuyama H, Nose K, Nakai H, Yoneda A, Kubota A et al. Physiological and clinical characteristics of gastroesophageal reflux after congenital diaphragmatic hernia repair. J Pediatr Surg 2010; 45: 2346-2350. 
38. Matsumura T, Arai M, Yonemitsu Y, Maruoka D, Tanaka T, Suzuki T et al. The traditional Japanese medicine Rikkunshito increases the plasma level of ghrelin in humans and mice. J Gastroenterol 2010; 45: 300-307.

39. Yakabi K, Kurosawa S, Tamai M, Yuzurihara M, Nahata M, Ohno S et al. Rikkunshito and 5-HT2C receptor antagonist improve cisplatin-induced anorexia via hypothalamic ghrelin interaction. Regul Pept 2010; 161: 97-105.

40. Halford JC, Harrold JA, Boyland EJ, Lawton CL, Blundell JE. Serotonergic drugs: effects on appetite expression and use for the treatment of obesity. Drugs 2007; 67: 27-55.

41. Vickers SP, Dourish CT, Kennett GA. Evidence that hypophagia induced by $d$-fenfluramine and $d$-norfenfluramine in the rat is mediated by 5 -HT2C receptors. Neuropharmacology 2001; 41: 200-209.

42. Vickers SP, Easton N, Webster LJ, Wyatt A, Bickerdike MJ, Dourish CT et al. Oral administration of the $5-\mathrm{HT} 2 \mathrm{C}$ receptor agonist, $\mathrm{mCPP}$, reduces body weight gain in rats over 28 days as a result of maintained hypophagia. Psychopharmacology 2003; 167 : 274-280.

43. Wang W, Danielsson A, Svanberg E, Lundholm K. Lack of effects by tricyclic antidepressant and serotonin inhibitors on anorexia in MCG 101 tumor bearing mice with eicosanoid-related cachexia. Nutrition 2003; 19: 47-53.

44. Makarenko IG, Meguid MM, Gatto L, Goncalves CG, Ramos EJ, Chen C et al. Hypothalamic 5HT1B-receptor changes in anorectic bearing rats. Neurosci Lett 2005; 376: 71-75.

45. Makarenko IG, Meguid MM, Gatto L, Chen C, Ugrumov MV. Decreased NPY innervation of the hypothalamic nuclei in rats with cancer anorexia. Brain Res 2003; 961: 100-108.

46. Wright DE, Seroogy KB, Lundgren KH, Davis BM, Jennes L. Comparative localization of serotonin1A, 1C, and 2 receptor subtype mRNAs in rat brain. $J$ Comp Neurol 1995; 351 : 357-373.
47. Heisler LK, Pronchuk N, Nonogaki K, Zhou L, Raber J, Tung L et al. Serotonin activates the hypothalamic-pituitary-adrenal axis via serotonin 2 C receptor stimulation. J Neurosci 2007; 27: 6956-6964.

48. Shintani F, Kanba S, Nakaki T, Nibuya M, Kinishita N, Suzuki E et al. Interleukin-1beta augments release of norepinephrine, dopamine, and serotonin in the rat anterior hypothalamus. J Neurosci 1993; 13: 3574-3581.

49. Chuang JC, Zigman JM. Ghrelin's roles in stress, mood, and anxiety regulation. Int J Pept 2010; id: 460549 .

50. Kamiji MM, Inui A. Neuropeptide $Y$ receptor selective ligands in the treatment of obesity. Endocr Rev 2007; 28: 664-684.

51. Date Y, Murakami N, Toshinai K, Matsukura S, Niijima A, Matsuo H et al. The role of the gastric afferent vagal nerve in ghrelin-induced feeding and growth hormone secretion in rats. Gastroenterology 2002; 123: 1120-1128.

52. Fujino K, Inui A, Asakawa A, Kihara N, Fujimura M, Fujimiya M. Ghrelin induces fasted motor activity of the gastrointestinal tract in conscious fed rats. J Physiol 2003; 550: 227-240.

53. Muroya S, Yada T, Shioda S, Takigawa M. Glucose-sensitive neurons in the rat arcuate nucleus contain neuropeptide Y. Neurosci Lett 1999; 264: 113-116.

Translational Psychiatry is an open-access journal published by Nature Publishing Group. This work is licensed under the Creative Commons Attribution-Noncommercial-Share Alike 3.0 Unported License. To view a copy of this license, visit http:// creativecommons.org/licenses/by-nc-sa/3.0/

Supplementary Information accompanies the paper on the Translational Psychiatry website (http://www.nature.com/tp) 